\title{
An OD-characterizable class of simple groups*
}

\author{
M. Akbari, X. Y. Chen, and A. R. Moghaddamfar
}

Communicated by I. Ya. Subbotin

\begin{abstract}
A BSTRACT. It is proved that nonabelian finite simple groups $S$ with $\max \pi(S)=37$ are uniquely determined by their order and degree pattern in the class of all finite groups.
\end{abstract}

\section{Introduction}

Throughout this note, all the groups under consideration are finite, and simple groups are nonabelian. Given a group $G$, the spectrum $\omega(G)$ of $G$ is the set of orders of elements in $G$. Clearly, the spectrum $\omega(G)$ is closed and partially ordered by the divisibility relation, and hence is uniquely determined by the set $\mu(G)$ of its elements which are maximal under the divisibility relation. One of the most well-known graphs associated with $G$ is the prime graph (or Gruenberg-Kegel graph) denoted by $\operatorname{GK}(G)$. The vertices of $\operatorname{GK}(G)$ are the prime divisors of $|G|$ and two distinct vertices $p$ and $q$ are joined by an edge (written by $p \sim q$ ) if $p q \in \omega(G)$. If $p_{1}<p_{2}<\cdots<p_{k}$ are all prime divisors of $|G|$, then we set

$$
\mathrm{D}(G)=\left(d_{G}\left(p_{1}\right), d_{G}\left(p_{2}\right), \ldots, d_{G}\left(p_{k}\right)\right),
$$

${ }^{*}$ This work was done during the second and third authors had a visiting position at the Department of Mathematical Sciences, Kent State University, USA. They would like to thank the hospitality of the Department of Mathematical Sciences of KSU. The second author was supported by the Fund for Young Key Teachers of Henan University of Technology, the Fund of Henan Administration of Foreign Experts Affairs, the Project of Henan Province (182102410049), and the NSFC (11926330, 11926326, 11971189, 11771356).

2010 MSC: 20D05, 20D06, 20D08.

Key words and phrases: OD-characterization of a finite group, prime graph, degree pattern, simple group. 
where $d_{G}\left(p_{i}\right)$ denotes the degree of $p_{i}$ in the prime graph $\operatorname{GK}(G)$. We call this $k$-tuple $\mathrm{D}(G)$ the degree pattern of $G$. In addition, we denote by $\mathrm{OD}(G)$ the set of pairwise non-isomorphic finite groups with the same order and degree pattern as $G$, and put $h(G)=|\mathrm{OD}(G)|$. Since there are only finitely many isomorphism types of finite groups of order $|G|$, $1 \leqslant h(G)<\infty$. Now, we have the following definition.

Definition 1. A group $G$ is said to be $k$-fold $O D$-characterizable if $h(G)=k$. As usual, a 1-fold OD-characterizable group is simply called $O D$-characterizable, and it is called quasi OD-characterizable if it is $k$-fold OD-characterizable for some $k>1$.

Notice that the OD-characterizability for simple groups $L_{2}(q)$ was proved in $[6,15]$. The OD-characterizability problem for alternating groups $A_{n}$ of degree $n(5 \leqslant n \leqslant 100)$ was investigated in [3] as well.

Given a prime $p, \mathfrak{S}_{p}$ stands for the set of nonabelian finite simple groups $S$ such that $p \in \pi(S) \subseteq\{2,3,5, \ldots, p\}$. Based on calculations in the computer algebra system GAP, the sets $\mathfrak{S}_{p}$ for which $p<10^{3}$ are determined in [14]. According to these results (see also [3]), if $S \in \mathfrak{S}_{37}$, then $S$ is isomorphic to one of the following simple groups:

$$
\begin{aligned}
& L_{2}(37), \quad U_{3}(11), \quad L_{2}\left(31^{2}\right), \quad S_{4}(31), \quad{ }^{2} G_{2}(27), \quad U_{3}(27), \\
& L_{2}\left(11^{3}\right), \quad G_{2}(11), \quad U_{4}(31), \quad A_{37}, \quad A_{38}, \quad A_{39}, \quad A_{40} .
\end{aligned}
$$

On the other hand, it has already been proved that the following simple groups are OD-characterizable: $L_{2}(37), L_{2}\left(31^{2}\right), L_{2}\left(11^{3}\right)$ [15], $U_{3}(11)$ [6], ${ }^{2} G_{2}(27)$ [6], $A_{37}, A_{38}, A_{39}, A_{40}$ [3]. So, in this note we will concentrate on the OD-characterizability problem for the rest of the groups, and the following is our main result.

Theorem A. The simple groups $S_{4}(31), U_{3}(27), G_{2}(11)$ and $U_{4}(31)$ are OD-characterizable.

Combining Theorem A and the above-envisaged results, we obtain the following corollary.

Corollary B. All simple groups in $\mathfrak{S}_{37}$ are $O D$-characterizable.

We introduce much more notation and definitions (notation used without further explanation is standard). Given a group $G$, we denote by $t(G)$ the maximal number of prime divisors of $G$ that are pairwise nonadjacent in $\operatorname{GK}(G)$, and by $t(r, G)$ the maximal number of prime divisors of $G$ containing $r$ that are pairwise nonadjacent in $\operatorname{GK}(G)$. Denote 
by $s(G)$ the number of connected components of $\mathrm{GK}(G)$ and by $\operatorname{GK}_{i}(G)$, $i=1,2, \ldots, s(G)$, the $i$ th connected component of $\mathrm{GK}(G)$. We also denote by $\pi_{i}=\pi_{i}(G), i=1,2, \ldots, s(G)$, the set of vertices of $i$ th connected component $\mathrm{GK}_{i}(G)$. If $G$ is a group of even order, then we put $2 \in \pi_{1}(G)$. It is now easy to see that the order of a group $G$ can be expressed as a product of some coprime natural numbers $m_{i}=m_{i}(G), i=1,2, \ldots, s(G)$, with $\pi\left(m_{i}\right)=\pi_{i}$, where $\pi\left(m_{i}\right)$ signifies the set of all prime divisors of $m_{i}$. The numbers $m_{1}, m_{2}, \ldots, m_{s(G)}$ are called the order components of $G$.

The sequel of this note is organized as follows. In Section 2, we recall some basic results, especially, on the spectra of certain finite simple groups, and they will help us find their degree patterns. Section 3 is devoted to the proof of our main result (Theorem A). Finally, we in Section 4 give a discussion of the relationship between two groups with the same order and degree pattern.

\section{Preliminaries}

Before proving our main result, we give several lemmas which will be required to determine the degree pattern of the groups under consideration.

Lemma 1 ([5]). Let $q=p^{n}$, where $p \neq 3$ is a prime. Then, we have

$$
\mu\left(S_{4}(q)\right)=\left\{\left(q^{2}+1\right) / 2,\left(q^{2}-1\right) / 2, p(q+1), p(q-1)\right\} .
$$

In particular, $\mu\left(S_{4}(31)\right)=\{480,481,930,992\}$.

Lemma 2 ([12]). If $q$ is a power of an odd prime $p$, then we have:

$$
\mu\left(U_{3}(q)\right)=\left\{\begin{array}{l}
\left\{q^{2}-q+1, q^{2}-1, p(q+1)\right\} \quad \text { if } q \neq \equiv-1 \quad(\bmod 3), \\
\left\{\frac{q^{2}-q+1}{3}, \frac{q^{2}-1}{3}, \frac{p(q+1)}{3}, q+1\right\} \quad \text { if } q \equiv-1 \quad(\bmod 3) .
\end{array}\right.
$$

In particular, $\mu\left(U_{3}(27)\right)=\{84,703,728\}$.

Lemma 3 ([10]). If $q$ is a power of a prime $p>5$, then we have:

$$
\mu\left(G_{2}(q)\right)=\left\{p(q-1), p(q+1), q^{2}-1, q^{2}-q+1, q^{2}+q+1\right\} .
$$

In particular, $\mu\left(G_{2}(11)\right)=\{110,111,120,132,133\}$.

Lemma 4 ([13]). Let $q$ be a power of an odd prime $p$. Denote $d=$ $\operatorname{gcd}(4, q+1)$. Then $\mu\left(U_{4}(q)\right)$ contains the following (and only the following) numbers: 
(1) $(q-1)\left(q^{2}+1\right) / d,\left(q^{3}+1\right) / d, p\left(q^{2}-1\right) / d, q^{2}-1$;

(2) $p(q+1)$, if and only if $d=4$;

(3) 9 , if and only if $p=3$.

In particular, $\mu\left(U_{4}(31)\right)=\{992,960,7215,7440,7448\}$.

Using Lemmas 1-4 and [14, Table 1], the required results concerning some simple groups in $\mathfrak{S}_{37}$ are collected in Table 1.

TABLE 1. The orders and degree patterns of some simple groups in $\mathfrak{S}_{37}$.

\begin{tabular}{lll}
\hline$S$ & $|S|$ & $\mathrm{D}(S)$ \\
\hline$S_{4}(31)$ & $2^{12} \cdot 3^{2} \cdot 5^{2} \cdot 13 \cdot 31^{4} \cdot 37$ & $(3,3,3,1,3,1)$, \\
$U_{3}(27)$ & $2^{5} \cdot 3^{9} \cdot 7^{2} \cdot 13 \cdot 19 \cdot 37$ & $(3,2,3,2,1,1)$ \\
$G_{2}(11)$ & $2^{6} \cdot 3^{3} \cdot 5^{2} \cdot 7 \cdot 11^{6} \cdot 19 \cdot 37$ & $(3,4,3,1,3,1,1)$ \\
$U_{4}(31)$ & $2^{16} \cdot 3^{2} \cdot 5^{2} \cdot 7^{2} \cdot 13 \cdot 19 \cdot 31^{6} \cdot 37$ & $(5,5,5,2,3,2,3,3)$ \\
\hline
\end{tabular}

Lemma 5 ([9]). Let $G$ be a finite group with $t(G) \geqslant 3$ and $t(2, G) \geqslant 2$, and let $K$ be the maximal normal solvable subgroup of $G$. Then the quotient group $G / K$ is an almost simple group, i.e., there exists a non-abelian simple group $P$ such that $P \leqslant G / K \leqslant \operatorname{Aut}(P)$.

Lemma 6 ([3]). Let $S$ be a simple group in $\bigcup_{5 \leqslant p \leqslant 97} \mathfrak{S}_{p}$. Then, we have $\pi(\operatorname{Out}(S)) \subseteq\{2,3,5\}$.

\section{Proof of Theorem A}

Before beginning the proof, we draw the prime graphs of the groups $S_{4}(31), U_{3}(27), G_{2}(11)$ and $U_{4}(31)$ in Figure 1.

Proof of Theorem A. Suppose first that $S$ is one of the simple groups $U_{3}(27), G_{2}(11)$ or $U_{4}(31)$. Let $G$ be a finite group such that $|G|=|S|$ and $\mathrm{D}(G)=\mathrm{D}(S)$. We have to prove that $G \cong S$. In all these cases we will prove that $t(G) \geqslant 3$ and $t(2, G) \geqslant 2$. Therefore, it follows from Lemma 5 that there exists a simple group $P$ such that $P \leqslant G / K \leqslant \operatorname{Aut}(P)$, where $K$ is the maximal normal solvable subgroup of $G$. In addition, we will prove that $P \cong S$, which implies that $K=1$ and since $|G|=|S|, G$ is isomorphic to $S$, as required. We handle every case singly.

(a) $S=U_{3}(27)$. Let $G$ be a finite group such that

$$
|G|=\left|U_{3}(27)\right|=2^{5} \cdot 3^{9} \cdot 7^{2} \cdot 13 \cdot 19 \cdot 37,
$$




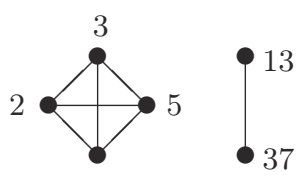

31

$\operatorname{GK}\left(S_{4}(31)\right)$

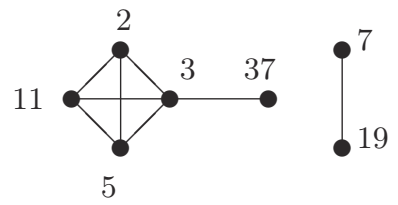

$\operatorname{GK}\left(G_{2}(11)\right)$

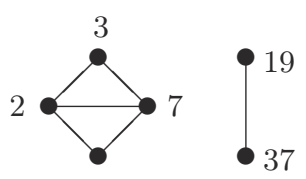

13

FIGURE 1. The prime graphs of some simple groups in $\mathfrak{S}_{37}$.

and

$$
\mathrm{D}(G)=\mathrm{D}\left(U_{3}(27)\right)=(3,2,3,2,1,1) .
$$

We now consider two cases $19 \sim 37$ and $19 \nsim 37$, separately.

(a.1) Assume first that $19 \sim 37$. In this case we immediately have that $\operatorname{GK}(G)=\operatorname{GK}\left(U_{3}(27)\right)$, and the hypothesis that $|G|=\left|U_{3}(27)\right|$ yields $G$ and $U_{3}(27)$ having the same set of order components. Now, by the Main Theorem in [2], $G$ is isomorphic to $U_{3}(27)$, as required.

(a.2) Assume next that $19 \nsim 37$. In this situation, there exists a prime $p \in \pi(G) \backslash\{19,37\}$ such that $\{p, 19,37\}$ is an independent set, since otherwise we get $d_{G}(19) \geqslant 2$ or $d_{G}(37) \geqslant 2$, which is impossible. This shows that $t(G) \geqslant 3$. Moreover, since $d_{G}(2)=3$ and $|\pi(G)|=6, t(2, G) \geqslant$ 2. Thus by Lemma 5 there exists a simple group $P$ such that $P \leqslant$ $G / K \leqslant \operatorname{Aut}(P)$, where $K$ is the maximal normal solvable subgroup of $G$. Let $\pi=\{7,13,19,37\}$. We claim that $K$ is a $\pi^{\prime}$-group. First of all, if $\{19,37\} \subseteq \pi(K)$, then a Hall $\{19,37\}$-subgroup of $K$ is an abelian group of order $19 \cdot 37$, and hence $19 \sim 37$, which is a contradiction. Now, assume that $\{p, q\}=\{19,37\}$ and $p$ does not divide the order of $K$ while $q \in \pi(K)$. Let $Q$ be a Sylow $q$-subgroup of $K$. By Frattini argument $G=K N_{G}(Q)$. Then, the normalizer $N_{G}(Q)$ contains an element of order $p$, say $x$. Now, $Q\langle x\rangle$ is an abelian group of order $p q$, and so $p \sim q$, again a contradiction. This shows that $\pi(K) \cap\{19,37\}=\varnothing$. With the similar arguments, we can verify that if $13 \in \pi(K)$, then 13 is adjacent to each of the three 
vertices 7,19 , and 37 , and this forces $d_{G}(13) \geqslant 3$, which contradicts the hypothesis. Finally, if $7 \in \pi(K)$, then again 7 is adjacent to each of the three vertices 13,19 , and 37 . Note, however, that the degree sequence of the subgraph GK $(G) \backslash\{7\}$ would be 3,2 , 1, which is impossible. Therefore, $K$ is a $\pi^{\prime}$-group. Since both $K$ and $\operatorname{Out}(P)$ are $\pi^{\prime}$-groups (Lemma 6), $|P|$ is divisible by $7^{2} \cdot 13 \cdot 19 \cdot 37$. Considering the orders of simple groups in $\mathfrak{S}_{37}$, we conclude that $P$ is isomorphic to $U_{3}(27)$. Therefore, $K=1$ and $G$ is isomorphic to $U_{3}(27)$. But then $\operatorname{GK}(G)=\operatorname{GK}\left(U_{3}(27)\right)$ and $19 \sim 37$, which is impossible.

The proof of the other cases is quite similar to the proof in the previous case, so we avoid here full explanation of all details.

(b) $S=G_{2}(11)$. Assume that $G$ is a finite group such that

$$
|G|=\left|G_{2}(11)\right|=2^{6} \cdot 3^{3} \cdot 5^{2} \cdot 7 \cdot 11^{6} \cdot 19 \cdot 37,
$$

and

$$
\mathrm{D}(G)=\mathrm{D}\left(G_{2}(11)\right)=(3,4,3,1,3,1,1) .
$$

We will consider two cases $7 \sim 19$ and $7 \nsim 19$, separately.

(b.1) First, suppose that $7 \sim 19$. In this case, it follows from the equality $\mathrm{D}(G)=\mathrm{D}\left(G_{2}(11)\right)$ that the prime graphs of $G$ and $S$ coincide. Thus, the hypothesis that $|G|=\left|G_{2}(11)\right|$ yields $G$ and $G_{2}(11)$ having the same set of order components. Now, by the Main Theorem in [7], $G$ is isomorphic to $S$, as required.

(b.2) Next, suppose that $7 \nsim 19$. In this case, on the one hand there exists a prime $p \in \pi(G) \backslash\{7,19\}$ such that $\{p, 7,19\}$ is an independent set, and so $t(G) \geqslant 3$. On the other hand, since $d_{G}(2)=3$ and $|\pi(G)|=6$, $t(2, G)=3$. Thus, by Lemma 5 , there exists a simple group $P$ such that $P \leqslant G / K \leqslant \operatorname{Aut}(P)$, where $K$ is the maximal normal solvable subgroup of $G$. Using similar arguments to those in the previous case, one can show that $K$ is a $\{7,19,37\}^{\prime}$-group and $G$ is isomorphic to $G_{2}(11)$. But then 7 is adjacent to 19 in $\operatorname{GK}(G)$, which is a contradiction.

(c) $S=U_{4}(31)$. Assume that $G$ is a finite group such that

$$
|G|=\left|U_{4}(31)\right|=2^{16} \cdot 3^{2} \cdot 5^{2} \cdot 7^{2} \cdot 13 \cdot 19 \cdot 31^{6} \cdot 37,
$$

and

$$
\mathrm{D}(G)=\mathrm{D}\left(U_{4}(31)\right)=(5,5,5,2,3,2,3,3) .
$$

First of all, we show that $t(G) \geqslant 3$. To this end, we will consider separately the two cases: $7 \sim 19$ and $7 \nsim 19$. If 7 is adjacent to 19 and to another vertex, say $p$, then the induced graph on $\pi(G) \backslash\{7,19, p\}$ is not complete, 
because we have only three vertices with degree $\geqslant 4$. Therefore, there are at least two nonadjacent vertices $r$ and $s$ in $\pi(G) \backslash\{7,19, p\}$. This shows that $\{7, r, s\}$ is an independent set in $\operatorname{GK}(G)$ and so $t(G) \geqslant 3$. If 7 and 19 are nonadjacent, then since $d_{G}(7)=d_{G}(19)=2$ there exists a vertex which is not adjacent to either of these two vertices, and again we conclude that $t(G) \geqslant 3$. Moreover, since $d_{G}(2)=5$ and $|\pi(G)|=8$, $t(2, G) \geqslant 2$. Thus by Lemma 5 there exists a simple group $P$ such that $P \leqslant G / K \leqslant \operatorname{Aut}(P)$, where $K$ is the maximal normal solvable subgroup of $G$. In addition, $K$ is a $\{7,19,37\}^{\prime}$-group. Indeed, as before, if $7 \in \pi(K)$ or $19 \in \pi(K)$, this would yield $\operatorname{deg}_{G}(7) \geqslant 3$ or $\operatorname{deg}_{G}(19) \geqslant 3$, which is not the case. Finally, if $37 \in \pi(K)$, then we obtain $\operatorname{deg}_{G}(37) \geqslant 4$, and thus we have a contradiction. Since both $K$ and $\operatorname{Out}(P)$ are $\{7,19,37\}^{\prime}$-groups (Lemma 6), $|P|$ is divisible by $7^{2} \cdot 19 \cdot 37$. Considering the orders of simple groups in $\mathfrak{S}_{37}$ yields $P$ isomorphic to $U_{4}(31)$. But then $K=1$ and $G$ is isomorphic to $U_{4}(31)$, because $|G|=\left|U_{4}(31)\right|$.

Next we concentrate on the simple group $S_{4}(31)$.

(d) $S=S_{4}(31)$. Suppose that $G$ is a finite group such that

$$
|G|=\left|S_{4}(31)\right|=2^{12} \cdot 3^{2} \cdot 5^{2} \cdot 13 \cdot 31^{4} \cdot 37,
$$

and

$$
\mathrm{D}(G)=\mathrm{D}\left(S_{4}(31)\right)=(3,3,3,1,3,1) .
$$

We distinguish two cases separately.

(d.1) Assume first that $13 \sim 37$. In this case we immediately have that $\operatorname{GK}(G)=\operatorname{GK}\left(S_{4}(31)\right)$, and since $|G|=\left|S_{4}(31)\right|$ we conclude that $G$ and $S_{4}(31)$ have the same set of order components. Now, by the Main Theorem in [1], $G$ is isomorphic to $S_{4}(31)$, as required.

(d.2) Assume next that $13 \nsim 37$. Let $\left\{p_{1}, p_{2}, p_{3}, p_{4}\right\}=\{2,3,5,31\}$. The prime graph $\operatorname{GK}(G)$ is depicted in Figure 2. Clearly, $t(G) \geqslant 3$, and since $d_{G}(2)=3$ and $|\pi(G)|=6, t(2, G) \geqslant 2$.

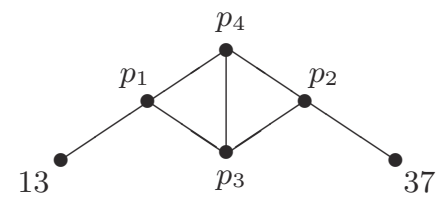

FIGURE 2. The prime graph of $G$.

Thus by Lemma 5 there exists a simple group $P$ such that $P \leqslant$ $G / K \leqslant \operatorname{Aut}(P)$, where $K$ is the maximal normal solvable subgroup of 
$G$. As before, one can show that $K$ is a $\{13,31,37\}^{\prime}$-group. Since $K$ and $\operatorname{Out}(P)$ are $\{13,31,37\}^{\prime}$-groups (Lemma 6), thus $|P|$ is divisible by $13 \cdot 31^{4} \cdot 37$. Considering the orders of simple groups in $\mathfrak{S}_{37}$ yields that $P$ is isomorphic to $S_{4}(31)$. But then $K=1$ and $G$ is isomorphic to $S_{4}(31)$, because $|G|=\left|S_{4}(31)\right|$. Therefore $\operatorname{GK}(G)=\operatorname{GK}\left(S_{4}(31)\right)$ which is disconnected, a contradiction.

This completes the proof of theorem.

\section{Some remarks}

Given a finite group $M$, suppose that $G$ is a finite group with (1) $|G|=|M|$ and $(2) \mathrm{D}(G)=\mathrm{D}(M)$. In most cases, it follows from the above conditions that they have the same order components. We denote by $\operatorname{OC}(G)$ the set of order components of $G$. The group $M$ is said to be characterizable by order component if, for every finite group $G$, the equality $\mathrm{OC}(G)=\mathrm{OC}(M)$ implies the group isomorphism $G \cong M$. It has already been shown that many simple groups are characterizable by order component (for instance, see $[1,2,7])$. Therefore, when under the conditions $|G|=|M|$ and $\mathrm{D}(G)=\mathrm{D}(M)$ we can conclude that $\mathrm{OC}(G)=\mathrm{OC}(M)$, and $M$ is characterizable by order component, it follows that $M$ is ODcharacterizable too. However, in the case when the prime graph of $M$ is connected, the group $M$ is not necessarily characterizable by order component, but it may be OD-characterizable. For instance, as we have seen in Theorem A, the simple group $U_{4}(31)$ is OD-characterizable, however all nilpotent groups (especially, abelian groups) of order $\left|U_{4}(31)\right|$ have the same order component, that is $\left|U_{4}(31)\right|$, which means that $U_{4}(31)$ is not characterizable by order component.

Given a nonnegative integer $n$, we set

$$
D_{n}(G)=\left\{p \in \pi(G) \mid d_{G}(p)=n\right\} .
$$

Since $\operatorname{GK}(G)$ is a simple graph, $D_{n}(G)=\varnothing$ for all $n \geqslant|\pi(G)|$. Some information on the prime graph of $G$ is obtained from $D_{n}(G)$ for some $n$. For instance, since $d_{G}(p)=0$ if and only if $\{p\}$ is a connected component of $\operatorname{GK}(G)$, we conclude that $\left|D_{0}(G)\right| \leqslant s(G) \leqslant 6$ (see [11]). On the other hand, if $D_{n-1}(G) \neq \varnothing$, where $n=|\pi(G)|, \operatorname{GK}(G)$ is connected, that is $s(G)=1$. Some examples of such groups are the symmetric groups $S_{m}$, for which $m-l_{m} \geqslant 2$, and alternating groups $A_{m}$, for which $m-l_{m} \geqslant 3$, where $l_{m}$ is the largest prime less than $m$. In [8, Theorem B], Suzuki proved that if $L$ is a finite simple group such that $\operatorname{GK}(L)$ is disconnected, then the connected component $\operatorname{GK}_{i}(L), i \geqslant 2$, is a clique (we recall that 
a clique is a set of vertices each pair of which is connected by an edge). As a matter of fact, this is true for all finite groups not only for finite simple groups. Hence, the prime graph of an arbitrary finite group $G$ has the following form:

$$
\mathrm{GK}(G)=\bigoplus_{i=1}^{s} \mathrm{GK}_{i}(G)=\mathrm{GK}_{1}(G) \oplus K_{n_{2}} \oplus \cdots \oplus K_{n_{s}}
$$

where $n_{i}=\left|\pi_{i}(G)\right|(2 \leqslant i \leqslant s)$ and $s=s(G)$. Thus, we conclude that $\left|D_{n_{i}-1}(G)\right| \geqslant n_{i},(2 \leqslant i \leqslant s)$. The sets $\pi_{i}(G), i=1,2, \ldots, s(G)$, for finite simple groups $G$ are listed in [4] and [11]. Under the conditions (1) and $(2)$, if there exists a vertex $p \in D_{0}(M)$, then $\pi_{i}(M)=\{p\}=\pi_{j}(G)$ for some $i, j$. This restriction helps us to determine the structure of $G$.

\section{References}

[1] A. Iranmanesh and B. Khosravi, A characterization of $C_{2}(q)$ where $q>5$, Comment. Math. Univ. Carolin., 43 (2002), 9-21.

[2] A. Iranmanesh, B. Khosravi and S. H. Alavi, A characterization of $\operatorname{PSU}_{3}(q)$ for $q>5$, Southeast Asian Bull. Math., 26 (2002), 33-44.

[3] R. Kogani-Moghaddam and A. R. Moghaddamfar, Groups with the same order and degree pattern, Sci. China Math., 55 (2012), 701-720.

[4] A. S. Kondratév, On prime graph components of finite simple groups, USSR-Sb., 67 (1990), 235-247.

[5] V. D. Mazurov, Recognition of the finite simple groups $S_{4}(q)$ by their element orders, Algebra Logic, 41 (2002), 93-110.

[6] A. R. Moghaddamfar, A. R. Zokayi and M. R. Darafsheh, A characterization of finite simple groups by the degrees of vertices of their prime graphs, Algebra Colloq., 12 (2005), 431-442.

[7] P. Nosratpur and M. R. Darafsheh, Characterization of the groups $G_{2}(q)$ for $2<q \equiv-1(\bmod 3)$ by order components, Sib. Math. J., 54 (2013), 883-893.

[8] M. Suzuki, On the prime graph of a finite simple group - an application of the method of Feit-Thompson-Bender-Glauberman, Groups and combinatorics - in memory of Michio Suzuki, Adv. Stud. Pure Math., 32 (Math. Soc. Japan, Tokyo, 2001), 41-207.

[9] A. V. Vasilév and I. B. Gorshkov, On the recognition of finite simple groups with a connected prime graph, Sib. Math. J., 50 (2009), 233-238.

[10] A. V. Vasilév and Staroletov, Recognizability of the groups $G_{2}(q)$ by the spectrum, Algebra Logic, 52 (2013), 1-14.

[11] J. S. Williams, Prime graph components of finite groups, J. Algebra, 69 (1981), 487-513.

[12] A. V. Zavarnitsin, Recognition of the simple groups $\mathrm{U}_{3}(q)$ by element orders, Algebra Logic, 45 (2006), 106-116. 
[13] A. V. Zavarnitsine, Exceptional action of the simple groups $\mathrm{L}_{4}(q)$ in the defining characteristic, Sib. Élektron. Mat. Izv., 5 (2008), 68-74.

[14] A. V. Zavarnitsine, Finite simple groups with narrow prime spectrum, Sib. Elektron. Mat. Izv., 6 (2009), 1-12.

[15] L. C. Zhang and W. J. Shi, OD-characterization of the projective special linear groups $L_{2}(q)$, Algebra Colloq., 19 (2012), 509-524.

\section{CONTACT INFORMATION}

Majid Akbari Department of Mathematics, Payame Noor

University, P.O. Box 19395-3697, Tehran, Iran E-Mail(s): majid.math@gmail.com

Xiaoyou Chen College of Sciences, Henan University of Technology, 450001, Zhengzhou, China

E-Mail(s): cxy19800222@163.com

Ali Reza Faculty of Mathematics, K. N. Toosi University of

Moghaddamfar Technology, P.O. Box 16765-3381, Tehran, Iran

E-Mail(s): moghadam@kntu.ac.ir

Web-page(s): https://wp.kntu.ac.ir/moghadam/

Received by the editors: 22.01.2018. 